\title{
Basics of research (Part 12): Qualitative research
}

Cheryl Thompson

University of Nebraska Medical Center, cbthompson@unmc.edu

B. Lee Walker

University of Utah

Tell us how you used this information in this short survey.

Follow this and additional works at: https://digitalcommons.unmc.edu/con_articles

Part of the Nursing Commons

\section{Recommended Citation}

Thompson, Cheryl and Walker, B. Lee, "Basics of research (Part 12): Qualitative research" (1998). Journal Articles: College of Nursing. 22.

https://digitalcommons.unmc.edu/con_articles/22

This Article is brought to you for free and open access by the College of Nursing at DigitalCommons@UNMC. It has been accepted for inclusion in Journal Articles: College of Nursing by an authorized administrator of DigitalCommons@UNMC.For more information, please contact digitalcommons@unmc.edu. 


\title{
Basics of Research (Part 12): Qualitative Research
}

\author{
Cheryl Bagley Thompson, PhD, RN, ${ }^{1}$ and B. Lee Walker, PhD, RN1
}

1. University of Utah College of Nursing, Salt
Lake City

Address for correspondence and reprints: Cheryl Bagley Thompson, PhD, RN, University of Utah College of Nursing, $10 \mathrm{~S}$. 2000 E. Front St., Salt Lake City, UT 84112

Key Words: nursing research, qualitative research

Copyright $\odot 1998$ by the Air Medical Journal Associates

$1067-991 \times / 98 / \$ 5.00+0$

Reprint no. 74/1/89231
When a researcher has questions about events or experiences for which litthe is known, designing a research study that will provide causal explanation or prediction may be difficult. In such cases, because little or no knowledge of variables exists that may be pertinent to the event or experience or how these variables may be related, a qualitative research study may be the most appropriate approach. Qualitative research examines events or experiences in context from the perspective of the individuals experiencing the phenomena. This approach allows the researcher to explore the depth and complexity of a phenomenon, identify and describe its components and their relationships, and develop a picture of the whole that can enhance and guide practice and future research.

A qualitative approach also is appropriate when an investigator seeks to generate new theory or reformulate ideas about a known phenomenon or process when some indication exists that current knowledge or theories may be incomplete or biased. ${ }^{1,2} \mathrm{~A}$ qualitative method also may be used to identify questions and develop instruments for quantitative research.

Qualitative research is appropriate to address such questions as, "What is going on here? How can I explain it?" or to describe how people live or cope with particular experiences. Such questions frequently arise in clinical settings. For example, if a researcher wants to understand how patients perceive the stress of transport or how parents cope with the information that their premature newborn may not survive the trip to the neonatal tertiary care center, a qualitative approach may be the method of choice.

\section{Comparison of Approaches}

Although the terms quantitative and qualitative are merely descriptions of two types of data (numbers and words, respectively), the methods associated with them approach the research process from different perspectives. Table 1 outlines some of these contrasts. The researcher using a quantitative approach seeks to explain causes and make predictions. ${ }^{2}$ Stemming from an assumption that a reality exists that may be discovered and manipulated, this researcher investigates the problem deductively, examining variables thought to be pertinent based on either existing theory or his or her own interpretation of the phenomenon. Attempts are made to control intervening variables arising from the particular research context to ensure generalizability to other situations or contexts. Data are numbers derived from subjects' responses to paper and pencil activities or from some objective measurement (e.g., laboratory analysis of blood, temperature measurements). Random selection of a large and representative sample from the population of interest is used to control bias. Throughout the process, the researcher strives to maintain the stance of an objective outsider.

The qualitative researcher, on the other hand, assumes the existence of 
multiple realities based on the belief that people construct meaning in relation to the world in which they live and that each individual reality is unique in some respect. Rather than attempt to control variables, all aspects of the problem are explored in depth, and any intervening variables in the process are considered part of the phenomenon. ${ }^{2}$ Description of or speculation about the phenomenon develops inductively, derived from participants' interpretation of events in the context in which these events are experienced. In research related to patient transport, participants may be patients, transport personnel, or family members. Data are collected primarily by interview but also may be derived from observation and supplemented by a variety of pictorial or written records.

\section{Unique Features \\ of Qualitative Research}

Several features related to roles (of both the researchers and study participants) and to the research design also differentiate qualitative from quantitative research. In qualitative studies, people who agree to take part are considered partners in the discovery and verification of knowledge, participants in the research process rather than "subjects." Participant and researcher interchange increases the sensitivity of each to the topic under study, making possible a fuller understanding and interpretation of the phenomena. Both the interaction be tween researcher and participant and the reactions and ideas of the researcher recorded as field notes and memos become part of the study data; in this way the final product incorporates both researcher and participant.

In qualitative studies, decisions regarding the selection and size of the sample differ markedly from those in quantitative studies. Participants in qualitative studies are selected because they are knowledgeable about or have experienced the phenomenon of interest and are able to share that knowledge with the researcher. In some approaches (e.g., grounded theory), ongoing participant selection is guided by information emerging from concurrent data analysis as part of the interviewing process and its analysis. The researcher may increase the sample based on theoretical (purpo-

\section{Table 1}

\section{Contrasts between}

\section{Quantitative and Qualitative Approaches}

\begin{tabular}{|c|c|c|}
\hline & Quantitative & Qualitative \\
\hline Purpose & $\begin{array}{l}\text { Explores causes, } \\
\text { makes predictions }\end{array}$ & $\begin{array}{l}\text { Aims to describe phenomenon } \\
\text { or generate theory }\end{array}$ \\
\hline Perspective & $\begin{array}{l}\text { Objectivity increased through } \\
\text { use of precise measurement }\end{array}$ & Subjective view of participants \\
\hline Sample & $\begin{array}{l}\text { Large, representative samples } \\
\text { Random selection of subjects } \\
\text { or random assignment to group }\end{array}$ & $\begin{array}{l}\text { Small samples } \\
\text { Purposive selection of participants } \\
\text { based on their experience }\end{array}$ \\
\hline Data & $\begin{array}{l}\text { Generated from responses } \\
\text { to questionnaires or some } \\
\text { objective measurement } \\
\text { (e.g., temperature) }\end{array}$ & $\begin{array}{l}\text { Consist of words (interviews, } \\
\text { diaries, other written documents) } \\
\text { or pictures or other artifacts in } \\
\text { which the significance has been } \\
\text { rendered into words }\end{array}$ \\
\hline Analysis & Statistical & Interpretive \\
\hline
\end{tabular}

\section{Table 2}

Phenomenology

Grounded Theory

Ethnography

Historical

sive) sampling, a process in which the researcher seeks to verify hunches or determine if emerging theory fits in different situations or with participants who have selected characteristics. In ethnography, initial participants are chosen because they can provide entry to a group or culture and can assist the researcher either by explaining activities and behavior that the researcher may have observed or by suggesting further informants who can provide the explanations the researcher seeks.

Because any qualitative approach generates enormous amounts of primarily verbal data, large sample sizes usually are not feasible. The number of participants needed can be estimated only just before data collection. As data collection progresses, sample size is determined by the criterion of "saturation," the point at which the researcher is obtaining the same data over and over again and obtaining new information is unlikely. The fact that saturation does occur supports the social/psychologic position that, although each individual is unique, patterns $d o$ exist and that people tend to make sense of their experiences in similar ways.

\section{Qualitative Methods}

Several research approaches are grouped under the heading of qualitative methods. Table 2 defines a few of these approaches. Some of these methods are rooted in theoretical and philosophical beliefs that direct inquiry and guide analysis (phenomenology, grounded the ory). Other approaches have developed as methods of inquiry in particular disci- 
plines (ethnography, historical research), and they also have rules that provide detailed guidance related to data generation and interpretation. Some ap proaches often discussed as qualitative methods probably are more accurately designated as data collection methods (focus groups) or guides to data management and analysis (content analysis, case study methods).

This arlicle briefly describes three of the most frequently encountered qualitative research approaches: grounded the ory, phenomenology, and ethnography. Discussion of each method is woven around research studies using that approach. References associated with each method provide more direction for readers interested in further exploration of the particular approach.

\section{Phenomenology}

Phenomenology seeks to understand and describe the experience of interest as it is lived, that is, from the perspective of the individual participant's subjective reality. Phenomenology has emerged from the works of such philosophers as Husserl, Kierkegaard, Heidegger, and Merleau-Ponty. ${ }^{3}$ The common theme in all phenomenologic approaches is the concern for human meaning and, in the case of nursing, how those meanings can be translated into information that will lead to more informed and sensitive patient care. ${ }^{3}$

The phenomenologic approach asks, "What is it like to have a certain experience?" or "What is the meaning of a particular experience to the individual living through that experience?" Swanson 4 used a phenomenologic approach to examine the care provided to neonates in a newborn intensive care unit by nurses, physicians, and parents. Swanson believed that all these individuals are responsible for caring for the infant. She asked them to respond to the question, "What is it like to be a provider of care in the NICU?" 4 Through her interviews, Swanson began to see that the care provided in the NICU consisted of managing responsibilities, caring, attaching, and avoiding bad outcomes within the context of the individual.

Data collection and analysis. Data for this example came primarily from interviews. Usually in qualitative studies, in- terviews are taped and transcribed verba tim. Such studies generate mounds of data, which must be reduced and synthesized into themes that communicate the essence of the experience. Phenomenologists' concern with "wholeness," however, makes them reluctant to condense data through any segmenting and reassembly. Although some segmentation usually occurs during analysis, most approaches to data analysis developed by phenomenologic researchers begin with a careful reading and rereading of the source material to capture the "essence" of an account. The aim is to understand meaning and actions. A number of researchers have developed guidelines for accomplishing this understanding while remaining true to the precepts of phenomenologic inquiry. Swanson 4 followed steps outlined by Giorgi ${ }^{5}$ and provided a description of the analytic process.

\section{Grounded Theory}

The purpose of grounded theory is to generate explanatory theory that provides further understanding of social and psychologic phenomena. The approach emerged from the social psychologic theory of symbolic interaction, which presents both a theory of human behavior and an approach to inquiring about group behavior. ${ }^{6}$ According to symbolic interaction theory, people create their reality by attaching meaning to situations. These meanings or beliefs are expressed through symbols, such as words, dress, hairstyles, objects of worship, etc. Although each person's reality is unique, groups share symbolic meanings, and these meanings form the basis for actions and interactions. In the process of interaction, meanings may change. Grounded theory research focuses on these symbols and interactions and how they change in particular situations to develop theories about social processes "grounded" or based in the lives of people experiencing the process.

Glaser and Strauss, ${ }^{7}$ two sociologists at the University of California at San Francisco, developed the specific techniques referred to as grounded theory method. In this approach, initial questions focus broadly on what is happening or how something occurred, then narrow to pursue specific paths or processes identified from interviews as collection of con- current data sharpens the research focus.

For example, Jenny and Logan ${ }^{8}$ used a grounded theory approach to describe how expert critical care nurses promote ventilator weaning. Three themes were embedded in the weaning process: knowing the patient, performing the work of weaning, and managing patient energy. 'These themes represented the major concerns that directed nurses' actions in promoting patient independence. The report is particularly valuable because it provides a description of the analysis and methods used to ensure trustworthy results.

Data collection and analysis. Several sources provide detailed guidance for the researcher whose study question calls for a grounded theory approach. ${ }^{68}$ Jenny and Logan's ${ }^{8}$ article provides an example of the constant comparative method used in grounded theory analysis. Data collection and analysis proceeded concurrently from the first interview. The process of developing categories began with the first interview, and, although these researchers do not mention it, questions can be added and the interview refocused based on emerging theory. The researchers recorded memos to document the progress of the ory development.

In the process of theory development, researchers often return to previous participants to verify interpretations, a tactic used by these researchers to increase the trustworthiness of their conclusions. In many studies, additional participants are selected and other data consulted as suggested by the emerging social psychologic process or theory, a step implied by Jenny and Logan 8 in their statement that this study was the first stage in developing a theory of the nursing process in ventilator weaning. In this study, as with many qualitative studies, most data were collected through written accounts and interviews with people directly involved with the process or phenomenon of interest. However, grounded theory studies also may use other data sources, such as written records or reports from individuals knowledgeable about but not necessarily directly experiencing the process.

\section{Ethnography}

Ethnography was developed by anthropologists to study cultures. Its purpose is 
to present a total picture of a defined group's daily life, including with beliers, patterns of activities, and meanings attached to these activities and behaviors. The researcher or participant observer becomes immersed in the everyday life of the culture being studied. He or she enters into that life to the extent possible, using multiple methods (interviews, observations, written records, pictures, artifacts) to understand how conditions of daily life and cultural patterns interact to influence the phenomena that is the focus of the research. The interest of the researcher influences both data collection and interpretation.

Data collection and analysis. In ethnographic research, data once again come primarily from interviews and observations but also may encompass diaries, pictures, and various cultural artifacts. Analytic methods tend toward descriptions aimed at providing an understanding of the group or culture being studied rather than toward theory building.

Using ethnography as her primary research method, Welch ${ }^{10,11}$ investigated the social environment of individuals who had experienced a traumatic injury. Welch followed 30 pcople for 14 months after their injury. During that time, she spoke not only with the victims but also their families, physicians, and nurses (all parts of the milieu influencing these individuals' perceptions and behaviors). In addition, Welch used a limited amount of participant observation as she collected data regarding the process of developing meaning from the experience of recovering from physical trauma.

All individuals, regardless of their recovery level, identified three basic stages in the process: crisis, healing, and recovery. Those who did not consider themselves recovered at the end of the study had experienced more severe injury, often leaving them with permanent disability or disfiguring scars. All the nonrecovered individuals experienced periods of depression during the study, and many identified the depression as a major impediment to their recovery. Themes identified in the ethnographic data that Welch recommended as potential intervention targets included a sense of abandonment after discharge, prolonged grief reaction, and the need for more sensitive treatment for physical symptoms, such as pain.

\section{Table 3}

\section{Data Reduction}

Participant 000

Causes of stress (self-doubt)

Some days coming into work I would be

petrified that I would do something wrong

during a transport.

Causes of stress (self-doubt)

I remember one particularly stressful patient.

This patient kept going into an arrhythmia, $\quad 76$

$\begin{array}{ll}\text { which I thought was } v \text { tach, but the } & 77\end{array}$

referring physician said wasn't. It was stressfull $\quad 78$

not knowing for sure and how best to treat it. $\quad 79$

I felt embarrassed in front of my teammate.

Causes of stress (physical harm)

One night just outside the city limits, we 120

unexpectedly hit a fog bank. The pilot 121

momentarily lost control as he lost the horizon. 122

For a short time I was afraid we were 123

going to go down.

Combining Methods: Triangulation

Methods sometimes are combined in a single study to confirm or clarify findings, a design approach known as method triangulation. The two main purposes of method triangulation are to increase a study's reliability and validity, which occurs when data generated by one method confirm the findings of another method, and to increase a study's comprehensiveness. Methods can be combined within a particular tradition, as demonstrated by Wilson and Hutchinson, ${ }^{12}$ who used two qualitative approaches (a Heideggerian phenomenologic approach and grounded theory) in a study of caregivers to discover meanings and ways of being while generating a conceptual framework (through grounded theory) helpful in planning interventions and guiding further research.

Methods also may be combined across traditions (quantitative with qualitative), although disagreement exists about the appropriateness of combining methods whose assumptions stem from such vastly different world views of the nature of reality. ${ }^{13}$ However, Sandelowski14 argues that including a qualitative component often enhances the clinical significance of study findings by helping explain why variables are linked by identifying the "actual configuration of events that led to specific outcomes in specific cases."

\section{Data Management and Analysis}

The data to be analyzed in qualitative studies consist primarily of words, but qualitative analysis calls for the same careful and critical scrutiny required in quantitative studies. The researcher's task is to synthesize an enormous amount of data, moving from concrete information to increasing levels of abstraction. Some guidance for data synthesis is provided by such researchers as Colaizzi ${ }^{15}$ and Giorgi ${ }^{5}$ for phenomenologic studies and by Glaser and Strauss ${ }^{7}$ for grounded theory.

However, the researcher, while adhering to the spirit of the qualitative approach guiding the study, has to find a usable system for data reduction and synthesis. Miles and Huberman ${ }^{16}$ identified three streams of activity common throughout most qualitative analyses: data reduction, data display, and conclusion drawing and verification. These activities overlap and are iterative or cyclical rather than separate and discrete steps in the analysis.

Data reduction consists of selecting, grouping, and summarizing data from transcripts, field notes, and other sources. This activity serves to sharpen and organize the data to assist in drawing final conclusions. As such, data reduction is an integral part of those conclusions because the decisions being made as to what segments to code, how to group them, or which pieces of data or patterns best summarize the various data portions are all analytic choices.

Table 3 provides an example of data reduction in which three segments of 


\section{Figure 1}

\section{Data Display}

Causes of stress

Internal

External

Self-doubt

Physical harm

text are taken from a discussion of the stress experienced by air transport personnel. The researcher has labeled individual segments as "causes of stress" and has underlined words and short phrases representing these strategies. The researcher further has noted that the "causes" identified include both internal (self-doubt) and external (physical harm) possibilities.

The purpose of the second activity, data display, is to develop a system that allows the researcher to view the reduced data in some organized fashion that helps compare groupings and sug. gests relationships. Reduced data may be displayed as networks or in columns, charts, or matrices. From this display, the researcher is able to see connections within the data and either draw conclusions or proceed with further analyses suggested by the display. Figure 1 is a display based on data from Table 3 .

The decisions regarding what to display (what goes in the rows and columns of a matrix, for example) or how to arrange elements hierarchically within a network are subjective analytical activities. The process of developing the data display also may lead the researcher back to the original sources to verify the context of a datum before assigning it to a particular category, an example of the iterative process of qualitative analysis that adds to its reliability.

Conclusion drawing, according to Miles and Huberman, ${ }^{16}$ occurs from the start of data collection as the researcher begins to note patterns and regularities. Ideally, the researcher remains open and skeptical, and final conclusions may not be drawn until data collection is complete, but these "hunches" often influence subsequent participant recruitment and data gathering. In Figure 1, the researcher indicates that a distinction ex- ists between internal and external causes of stress. This beginning data display provides a framework for further analysis that may or may not support the distinction depicted in Figure 1.

Conclusions also must be verified as analysis proceeds. Verification may involve returning to the interview data or field notes to determine if a conclusion actually is rooted in the data, or it may involve review and discussion with colleagues to develop consensus regarding the conclusions. Ideally, the researcher also returns to talk with at least some of the participants to verify that they recognize the processes described or that the narrative does capture the essence of their experience. This process of verification is an integral part of the evaluation of any qualitative study.

A number of computer programs have been developed that offer assistance with data management tasks by efficiently sorting, storing, and retrieving data segments while also allowing the researcher to move between grouped segments and the original context of each. 17 Some of these programs also assist in the development of a data display.

Although computer programs provide assistance in data management, the researcher must make the data reduction and display decisions, and only the researcher can draw final the conclusions.

\section{Evaluating Qualitative Research}

Quantitative studies are judged on criteria related to reliability and validity. However, because qualitative approaches have different rules regarding aims, evidence, inference, and verification, quantitative criteria are not appropriate for evaluating qualitative studies. ${ }^{18}$ Lincoln and Guba $^{19}$ proposed that four factors be used to assess the rigor of qualitative studies: credibility, transferability, de- pendability, and confirmability.

Credibility addresses the truth value of a study and occurs when the descriptions or interpretations presented are recognized immediately by people who have had that experience. These "member checks" were used in the Jenny and $\operatorname{Logan}^{8}$ study as one evidence of their findings' credibility. Results of a study are also credible when other researchers or readers, after having read the study, can recognize the experience when it occurs.

Transferability is achieved when findings can "fit" samples and settings beyond that of the particular study. Researchers may address this concern by recruiting a sample that varies in age, educational level, and role activities in an effort to make their findings applicable to as diverse a group as possible.

Dependability (or consistency) refers to the stability of the findings and the ability to track variance in the data over time. Lincoln and Guba ${ }^{19}$ proposed that auditability be the criterion for evaluating the consistency of qualitative findings. To meet this criterion, the qualitative researcher needs to keep a written record detailing and justifying what actually was done at each step in the research process. The record needs to be complete enough so that another researcher could arrive at similar conclusions by following this "decision trail." Jenny and Logan ${ }^{8}$ detail their efforts to ensure the dependability of their findings by having two researchers code the interviews and by their extensive use of memos and diagrams documenting the progression of the developing theory.

Finally, confirmability is the criterion Lincoln and Guba ${ }^{19}$ proposed to evaluate neutrality. Because the qualitative researcher is, in a sense, a part of the final product, total objectivity is not possible. Only when the results can be confirmed by others not involved in the research is it possible to verify that the findings are not simply the researcher's perception alone.

Confirmability is achieved when auditability, creditability, and fit are established. ${ }^{18}$ However, Sandelowski ${ }^{14}$ cautions qualitative researchers to avoid concentrating too much on cookbook methods to demonstrate research validity and to focus instead on the "art of their work." The purpose of the research 
report is not to defend how a qualitative study measures up to the standards of the quantitative paradigm but rather to present the unique knowledge stemming from the study in a manner that enhances understanding and practice.

\section{Ethical Issues}

Protecting participants is an important consideration in any research and includes providing individuals with enough information about the study to enable them to make an informed decision about participation. However, the personal, subjective nature of such research often makes it difficult to anticipate what may arise in the course of an interview or observation. Munhall ${ }^{20}$ recommends that consent in qualitative studies be an ongoing transaction in which researchers and participants revisit the decision to take part in the study as unforeseen events or consequences arise.

The investigator also must consider the impact that participation in a study might have on informants and must carefully weigh the value of the research against any potential harm. The researcher should strive to give participants a full understanding of how sharing emotionally laden information may affect them and must have a plan in place to care for participants who encounter psychologic trauma related to sharing their experiences.

The researcher also has the obligation to protect participant privacy. In studies with a small number of participants, results must be reported in such a manner that individual respondents cannot be identified.

\section{Summary}

Although both the assumptions and methods of qualitative and quantitative approaches in nursing research are different, both have the goal of furthering the scientific basis for practice. A variety of qualitative approaches are available, and which approach to use depends on the purpose of the research. In general, qualitative investigations address broad questions related to description, discovery, or theory building, and, as a consequence, the researcher is concerned with the entire context surrounding the phenomenon of interest rather than concentrating on specific variables thought to influence that phenomenon.

The type of data collected and the methods of analysis differ, but qualitative research demands the same careful attention to selecting a design appropriate to answer the research question and the same assurance of rigor in conducting the research and interpreting the results as is required in quantitative studies. When these issues are thoroughly addressed, the clinician has a basis for judging both the accuracy and the applicability of qualitative research findings.

\section{References}

1. Burns N, Grove SK The practice of nursing research: conduct, critique, and utilization. 2nd ed. Philadelphia: WB Saunders; 1997.

2. Morse JM, Field PA Qualitative research methods for health professionals. 2nd ed. Thousand Oaks (CA): Sage; 1995.

3. Boyd CO. Phenomenology: the method. In: Munhall PL, Boyd CO, editors. Nursing research: a qualitative approach. 2nd ed. New York: National League for Nursing; 1993. p. 99 132.

4. Swanson KM. Providing care in the NICU: sometimes an act of love. Adv Nurs Sci 1990;13:60-73.

5. Giorgi A. Psychology as a human science: a phe nomenologically based approach. New York: Harper \& Row; 1970.

6. Chenitz WC, Swanson JM. From practice to grounded theory: qualitative research in nursing. Menlo Park (CA): Addison-Wesley; 1986.

7. Glaser BG, Strauss AL. The discovery of grounded theory: strategies for qualitative research. New York: Aldine De Gruyter; 1967.
8. Jenny J, Logan J. Promoting ventilator independence: a grounded theory perspective. Dim of Crit Care Nurs 1994;13(1):29-37.

9. Stern PN. Using grounded theory method in nursing research. In: Leininger MM, editor. Qualitative research methods in nursing. Orlando (FL): Grune \& Stratton; 1985. p. 149 60.

10. Welch M. Trauma recovery: an ethnography [doctoral dissertation]. The University of Connecticut; 1990.

11. Weich M. Clients' experiences of depression during recovery from traumatic injury, Clin Nurse Spec 1995;9(2):92-8.

12. Wilson HS, Hutchinson SA. Triangulation of qualitative methods: Heideggerian hermeneutics and grounded theory. Qualitative Health Research 1991;1:263-76.

13. Boyd $\mathrm{CO}$. Combining qualitative and quantitative approaches. In: Munhall PL, Boyd CO, editors. Nursing research: a qualitative approach. 2nd ed. New York: National League for
Nursing; 1993. p. 454-75.

14. Sandelowski M. To be of use: enhancing the utiity of qualitative research. Nurs Outlook 1997;45:125-32.

15. Colaizzi PF. Psychological research as the phe nomenologists view it. In: Valle $\mathrm{R}$, King $\mathrm{M}$, editors. Existential phenomenological alternatives for psychology. New York: Oxford University Press; 1975. p. 48-71.

16. Miles MB, Huberman AM. Qualitative data analysis. 2nd ed. Thousand Oaks (CA): Sage; 1994.

17. Weitzman EA, Miles MB. Computer programs for qualitative data analysis. Newbury Park (CA): Sage; 1994.

18. Sandelowski $M$. The problem of rigor in qualita tive research. Adv Nurs Sci 1986;8(3):27-37.

19. Lincoln YS, Guba EG. Naturalistic inquiry. Beverly Hills (CA): Sage; 1985.

20. Munhall PL. Ethical considerations in qualitative research. West J Nurs Res 1988;10(2):150-62. 